\title{
Steuerrechtfertigung und Steuergerechtigkeit bis zur Mitte des 17. Jahrhunderts
}

\subsection{Anfänge einer generellen Steuerlehre}

Ende des 16. und Anfang des 17. Jahrhunderts stieg die Zahl der Streitigkeiten über Steuern stark an, gleichzeitig intensivierte sich die wissenschaftliche Auseinandersetzung zur Staatsfinanzierung und zu Steuern. An die Stelle der älteren Ablehnung jeglicher Steuern trat eine generelle Steuerrechtfertigungslehre. ${ }^{151}$ Leitbild war dabei das wohlgeordnete Gemeinwesen mit einer sparsamen Verwaltung, einer aktiven Wirtschaft und der durch die Obrigkeit garantierten Rechtswahrung. ${ }^{152}$ Die landesherrlichen Einkünfte wurden erstmals ausführlich in den ersten Schriften und Monografien zur Legitimation der Fürstensouveränität behandelt. ${ }^{153}$ Sie bildeten den Auftakt für eine rasch zunehmende Finanzliteratur. ${ }^{154}$

Die Autoren dieser Zeit konnten auf wissenschaftliche Vorarbeiten kaum zurückgreifen, sie verfolgten mit ihren Schriften das Ziel, Fürsten und andere Herrscher durch Idealbilder vom „guten“ Regenten zum „rechten Handeln“ anzuleiten.

151 Schwennicke, Andreas: „Ohne Steuer kein Staat“, S. 101.

152 Stolleis, Michael: Pecunia nervus rerum. Zur Staatsfinanzierung in der frühen Neuzeit, Frankfurt am Main 1983, S. 87.

153 Schulz, Hermann: Das System und die Prinzipien der Einkünfte im werdenden Staat der Neuzeit, dargestellt anhand der kameralwissenschaftlichen Literatur (1600-1836), Berlin 1982, S. 25-28.

154 Schwennicke, Andreas: „Ohne Steuer kein Staat“, S. 112. 
Gegen Ende des 16. Jahrhunderts veränderten sich die Vorschläge über Grundsätze des Regierens und Verwaltens und führten zu neuen Legitimationslehren von der Souveränität des Staates bzw. des Regenten. ${ }^{155}$

Schon in der vor- und frühkameralistischen Literatur wird Jean Bodin (15301596) erwähnt, der sich in seiner „Respublica“ $(1583,1592)$ offen zu „pecunia nervus rerum" bekannte und damit den Standpunkt vertrat, dass die politische Macht von Herrscher und Staat nur durch eine gute Finanzlage erlangt und vermehrt werden kann. ${ }^{156}$ Er erhob die Allgemeinheit der Steuerpflicht zur Voraussetzung der Besteuerung überhaupt. ${ }^{157}$ Sein Grundgedanke war von der Vorstellung getragen, dass alle Besteuerung der Untertanen legitimiert sein müsse; damit lehnte er die Verwendung der Gelder einerseits für den Privatnutzen des Fürsten, andererseits für das Privatwohl der Untertanen ab. ${ }^{158}$

Weiter als Bodin ging ein paar Jahre später Theophilus Ellychnius (1623) bei seiner Gliederung der Einnahmen des Fürsten, in der ersten deutschsprachigen Schrift, die sich ausschließlich mit den landesherrlichen Einkünften befasste. ${ }^{159}$ Andere Autoren stellten die fürstlichen Einkünfte unter verschiedenen Gesichtspunkten dar; dabei traten zunehmend Überlegungen zu den rechtlichen Voraussetzungen und wirtschaftlichen Auswirkungen in den Vordergrund. Die Autoren wendeten

155 Schulz, Hermann: Das System und die Prinzipien der Einkünfte, S. 26-28.

156 Ebd, S. 29; Mayer, Theodor: Geschichte der Finanzwirtschaft und Finanzwissenschaft vom Spätmittelalter bis zum Ende des 18. Jahrhunderts, in: Gerloff, Wilhelm/Meisel, Franz (Hrsg.): Handbuch der Finanzwissenschaft 1, Tübingen 1926, S. 210-244, S. 238 f.

157 Schulz, Hermann: Das System und die Prinzipien der Einkünfte, S. 70.

158 Stolleis, Michael: Pecunia nervus rerum, S. 90.

159 Theophilus Ellychnius unterscheidet (1) Einkünfte ohne Belastung der Untertanen: Erhalt und Pflege der Allmenden; Reduktion der Staatsausgaben; (2) spontane Dotationen an den Fürsten aus freiem Willen; (3) Einkünfte aus dem Transit von Fremden und Ausländern; Geleit-, Ein- und Ausfuhrzölle; (4) legitime Einkünfte von Untertanen: Kopfsteuern, Vermögensteuern, Notsteuern; besondere Einnahmen wie Konfiskationen; Zoll, Weg- und Lagergeld insbesondere auf Luxusgüter; Geldstrafen; Zinsen und Gefälle aus landwirtschaftlicher und gewerblicher Produktion der Untertanen; Anleihen; ausnahmsweise Finanzhilfen von der Kirche; Erlaubnisgelder für bestimmte Bauformen; (5) eher diskutable Einkünfte: Staatsbetriebe; Geldhandel; Abgaben für Geburt, Heirat und Beerdigung der Untertanen; Rekognitionsgelder bei Herrschaftsantritt; Gerichtsgebühren; Verkauf von staatseigenen Gütern; (6) „unlöbliche und unzimliche Stuck“: missbräuchlicher obrigkeitlicher Monopolhandel und andere Machenschaften; vgl. Körner, Martin: Steuern und Abgaben in Theorie und Praxis im Mittelalter und in der frühen Neuzeit, in: Schremmer, Eckart (Hrsg.): Steuern, Abgaben und Dienste vom Mittelalter bis zur Gegenwart. Referate der 15. Arbeitstagung der Gesellschaft für Sozial- und Wirtschaftsgeschichte vom 14. bis 17. April 1993 in Bamberg, Stuttgart 1994, S. 53-76, S. 61 
sich hin zu rein rationalen Geldbeschaffungsmethoden, zur verstärkten empirischen Fundierung der Wirtschaftspolitik und zum Aufbau eines effektiven Steuersystems. ${ }^{160}$

In diesem Sinn wird in der Forschungsliteratur immer wieder auf Georg Obrecht $(1547-1612)^{161}$ verwiesen, der der Frage nachging, auf welche Weise die Einkommen eines Herrschers billig vermehrt werden können, um damit große und nützliche Ausgaben zu erledigen. ${ }^{162}$ Dabei setzte Obrecht im Zusammenhang von Finanzen und Staatsräson folgende Akzente: Zunächst ging es ihm um die fast vollständige Identifizierung von Staatswohl und Staatsfinanzierung, dann um die juristisch relevanten Beziehungen zwischen "Justitia" und "Necessitas", wobei jedoch die Belastung der Untertanen durch Steuern begrenzt sein soll. Außerdem begründete er die Bevorzugung der gestaffelten Vermögensteuer vor der starren Kopfsteuer rechtlich und konstatierte, dass aus Gerechtigkeitserwägungen die armen Leute nicht belastet werden dürfen.

Mit seinen Vorschlägen nimmt er einen wichtigen Platz in der gesamten Entwicklung ein, die auf eine Stärkung des Absolutismus durch unabhängige Finanzquellen hinauslief, ohne von den Ständen abhängig zu sein. ${ }^{163}$

Das Steuerbewilligungsrecht der Stände besaß im frühen 17. Jahrhundert seine unbestreitbare Gültigkeit, da es gewachsenes, praktiziertes und damit gültiges Recht war. ${ }^{164}$ Allerdings vertraten die meisten Autoren auch die Ansicht, dass in Notfällen die Zustimmung der Stände entfallen könnte, ${ }^{165}$ es sei denn, der Herrscher kann keinen legitimen Grund für seine Steuerforderung vorbringen, wenn er z. B. einen ungerechten Krieg führen will. ${ }^{166}$

Der wachsende Finanzbedarf des frühmodernen Staates fand auch in der juristischen Steuerliteratur seinen Niederschlag: Jakob Bornitz (1560-1625), Christoph Besold (1577-1638), Kaspar Klock (1583-1655) und Hermann Conring (1606-1681) zählen zu den bekanntesten Autoren. Aus ihrer praktischen Tätigkeit heraus erschloss sich ihnen die steigende Bedeutung der Geldeinkünfte für das Werden und Wachsen des Staates. ${ }^{167}$

160 Stolleis, Michael: Pecunia nervus rerum, S. 81.

161 Wilke bezeichnet Georg Obrecht als den ersten Deutschen, der steuerliche Fragen wissenschaftlich zu behandeln unternahm; Wilke, Gustav: Die Entwicklung der Theorie des staatlichen Steuersystems in der deutschen Finanzwissenschaft des 19. Jahrhunderts, in: Finanz-Archiv 38 (1921), S. 1-108, S. 6.

162 Schulz, Hermann: Das System und die Prinzipien der Einkünfte, S. 41.

163 Stolleis, Michael: Pecunia nervus rerum, S. $82 \mathrm{f}$.

164 Schulz, Hermann: Das System und die Prinzipien der Einkünfte, S. 55 f.

165 Stolleis, Michael: Pecunia nervus rerum, S. 95; Mayer, Theodor: Geschichte der Finanzwirtschaft und Finanzwissenschaft, S. 240.

166 Schwennicke, Andreas: „Ohne Steuer kein Staat“, S. 144 f.

167 Schulz, Hermann: Das System und die Prinzipien der Einkünfte, S. $44 \mathrm{f}$. 
„Pecunia nervus rerum“, die sprichwörtliche Wendung, das Geld sei der Nerv aller Dinge - und vor allem der Nerv des Krieges -, reichte bis in die Antike zurück. Aber erst mit einem durch Bodin vermittelten Bild gewannen die Sätze „pecunia nervus rerum“ und „pecunia nervus belli“ eine solche Beliebtheit, dass zu vermuten ist, dass hierin ein wesentlicher Punkt des Selbstverständnisses der Zeit gesehen werden kann. Ihr massenhaftes Vorkommen beweist, wie sehr man vom Wahrheitsgehalt dieser Sätze überzeugt war. ${ }^{168}$ Die Gründe für die Verbreitung dieser Maximen lassen sich wie folgt zusammenfassen: ${ }^{169}$ Die wichtigste Ursache war der fundamentale Wandel der mittelalterlichen Ökonomie, der Übergang von der Natural- zur Geldwirtschaft. Die neu empfundene Macht des Geldes zeigte sich im Wesentlichen bei der Finanzierung der Kriege und der großen politischen Machtkämpfe, und parallel zur Ausweitung der Staatsaufgaben setzte eine Ausweitung und Bürokratisierung des Staatsapparates ein, in denen man den Übergang zum „modernen Staat“ erblickt. Ende des 16. Jahrhunderts glückte im Deutschen Reich der Aufbau einer bescheidenen Reichsfinanzverwaltung. ${ }^{170}$ Diese hatte die Aufgabe, die Matrikularbeiträge von den Reichsgliedern einzutreiben, was sich als nicht so einfach erwies, denn die Behörde verfügte lediglich über einen Personalund Sachmitteletat von 30 Beamten und 300 Federkielen. ${ }^{171}$

Die oben genannten Autoren der juristischen Steuerliteratur haben die enge Beziehung zwischen dem Leitsatz „pecunia nervus rerum“, der Konzentration und Intensivierung der Staatstätigkeit und ihrer Abhängigkeit von der Finanzierbarkeit besonders deutlich gesehen. ${ }^{172}$ Steuer und Staat bedingten sich wechselseitig, Staatsbildung und Finanzentwicklung gingen Hand in Hand. ${ }^{173}$

Bornitz hat jene später immer wiederholte Unterscheidung zwischen guter und schlechter Staatsräson eingeführt. ${ }^{174}$ Besold unterschied wie Bornitz die gute Staatsräson von der schlechten und bejahte die bei allen Staatsräson-Schriftstellern zentrale Frage, ob verwerfliche Mittel zu einem guten Zweck erlaubt seien. Bezeichnend ist, dass für Besold außerordentliche Auflagen einer ausführlichen Rechtfertigung und einer rechtlichen „causa“ bedürfen. ${ }^{175}$ Daneben trat auch bei Besold die Idee der Ge-

168 Stolleis, Michael: Pecunia nervus rerum, S. 65.

169 Ebd., S.68f.

170 Homburg, Stefan: Allgemeine Steuerlehre, S. $32 \mathrm{f}$.

171 Ebd., S.32f.

172 Stolleis, Michael: Pecunia nervus rerum, S. $71 \mathrm{f}$.

173 Ullmann, Hans-Peter: Der deutsche Steuerstaat. Geschichte der öffentlichen Finanzen vom 18. Jahrhundert bis heute, München 2005, S. 13.

174 Stolleis, Michael: Pecunia nervus rerum, S. 84.

175 Mayer, Theodor: Geschichte der Finanzwirtschaft, S. 240. 
genseitigkeit in den Vordergrund, der Staat dürfe dann Leistungen fordern, wenn er auch seinen Bürgern ein Äquivalent in Form von gemeinnützigen Leistungen bietet. Für Besold war dies die Gegenleistung für Freiheit und Sicherheit, den Ausbau der Infrastruktur, die Garantie des Geldwertes und einer wirksamen Justiz. ${ }^{176}$

Als ungewöhnlich umfassendes und reifes Werk wird in der Forschungsliteratur $^{177}$ die Dissertation des späteren kaiserlichen Pfalzgrafen, Syndicus der Stadt Braunschweig und Kanzlers von Minden, Hildesheim sowie der Grafschaft Stolberg, Kaspar Klock, hervorgehoben. Mit seiner im Jahr 1608 verteidigten Dissertation, die er zu dem im 1634 erschienenen Werk „Tractatus de contributionibus“ umarbeitete, war Klock der wichtigste Autor des 17. Jahrhunderts in Steuerfragen. Die Bedeutung seiner Werke lässt sich an der Anzahl der Neuauflagen bis in die Mitte des 18. Jahrhunderts ablesen. ${ }^{178}$

Klock wird in unserer Zeit oft als Pionier der Finanzwissenschaft und als wichtigster Begründer der deutschen Steuerlehre benannt, die die Grundlage für die Smith'sche Finanzwissenschaft legte. ${ }^{179}$ Er suchte nach einer gerechten, auf Leistung beruhenden, ohne großen Verwaltungsaufwand zu überwachenden Form der Besteuerung. Er musste, da es dafür eine reine Theorie und scharfe Begriffe noch nicht gab, das Ziel mit Hilfsvorstellungen beschreiben wie z.B. „wie die Leistung, so die Besteuerung". Klock näherte sich so den Prinzipien der Leistungsfähigkeit und der progressiven Besteuerung. ${ }^{180}$

Das Gleichheitsprinzip stützte er auf das Naturgesetz und plädierte dafür, die Steuervorrechte des Adels und der Geistlichkeit zu vermindern. ${ }^{181}$ Aus ethischen Gründen forderte er auch die Steuerbefreiung des Existenzminimums und warnte vor indirekten Steuern, da diese vor allem die Armen belasteten. Da die Mehrheit der Kameralisten rein fiskalisch dachte und höchstens aus Sorge um die Schatzkammer für eine Steuerermäßigung eintrat, war eine solche Sichtweise durchaus unüblich. Bei Klock tritt schon der Gedanke an die Einkommensteuer zutage, da er die Steuern auf Ertrag abwerfende Güter beschränken wollte. Er forderte die Anlage

176 Stolleis, Michael: Pecunia nervus rerum, S. 89.

177 Homburg, Stefan: Allgemeine Steuerlehre, S. 35; Schwennicke, Andreas: „Ohne Steuer kein Staat", 114 ff.; Stolleis, Michael: Pecunia nervus rerum, S. 90 f.; Wilke, Gustav: Die Entwicklung der Theorie, S.7.

178 Schwennicke, Andreas: „Ohne Steuer kein Staat“, S. 115.

179 Schefold, Bertram: Kaspar Klock. Der Vater der guten Staatsfinanzen, in: Frankfurter Allgemeine Zeitung vom 25.01.2014, S. 1-6; auf: http:/www.faz.net/aktuell/wirtschaft/ wirtschaftswissen/die-weltverbesserer/kaspar-klock-der-vater-der-guten-staatsfinanzen-12769341.html (letzter Zugriff: 28.02.2017).

180 Ebd., S. 1.

181 Mayer, Theodor: Geschichte der Finanzwirtschaft, S. 240. 
von Katastern zur Feststellung der Leistungsfähigkeit des einzelnen Objektes; er war auch einer der Ersten, die zwischen Roheinkommen und Reineinkommen sauber trennten, indem er Werbungskosten zulassen wollte. ${ }^{182}$

Klock zeigte, dass sich im Wesen der staatlichen Finanzen der Länder der Welt Geschichte und Kultur ausdrücken und dass die Besteuerung dem Entwicklungsstand angepasst werden muss. So führte er z.B. aus, dass in den reichen Niederlanden indirekte Steuern eher akzeptabel als in armen Ländern waren, weil die Löhne unter geregelten Verhältnissen das Existenzminimum garantierten. Im Elend Deutschlands nach dem Dreißigjährigen Krieg hatte eine Überlast der indirekten Steuern jedoch Hunger zur Folge. ${ }^{183}$ Klock schrieb aber ebenso über die sinnvolle Verwendung der Finanzen, nicht für fürstliche Verschwendung, sondern um das Gemeinwohl zu fördern; der Reichtum aller fördere dann auch den Glanz des Hofes. ${ }^{184}$

Hermann Conring erörterte im Einzelnen Fragen der Steuergerechtigkeit. ${ }^{185}$ Er bevorzugte die gestaffelte Vermögen- vor der Kopfsteuer und wies auch auf Gebote der politischen Klugheit der Lastenverteilung des Herrschers hin. Er lehnte die Besteuerung von Lebensmitteln ab, forderte sie hingegen für Luxuswaren. Schließlich behandelte er die Vermehrung und Erhaltung des Staatsschatzes; keineswegs sollte die Vermehrung des Staatsschatzes um jeden Preis verfolgt werden. Das Unabänderliche müsse man den Untertanen wenigstens gerecht erscheinen lassen: „justissimum est, quod necessarium“, und die "necessitas“ gebietet auch die Begrenzung. Gerechtigkeit und politische Klugheit erforderten also überflüssig gewordene Lasten auch wieder abzuschaffen. ${ }^{186}$

Zusammenfassend kann festgestellt werden, dass jeder der Autoren, Obrecht, Bornitz, Besold, Klock und Conring, auf eine soziale Harmonie hin argumentierte. Allen Autoren war klar, dass man systematisch nach gleichmäßig fließenden Einnahmequellen suchen müsse, dabei jedoch die Gebote der politischen Klugheit und des Rechts nicht verletzen dürfe. ${ }^{187}$

Zwischen 1590 und 1670 bahnte sich unverkennbar ein Wandel der bis dahin vorherrschenden Meinungen in mehreren Punkten an: Erstens wird die Steuer als dauerhaftes Instrument akzeptiert. Einwendungen richten sich gegen die Höhe, den Maßstab (Vermögen- oder Kopfsteuer) und gegen die Art der Erhebung (direkte oder

182 Homburg, Stefan: Allgemeine Steuerlehre, S. 35.

183 Schefold, Bertram: Kaspar Klock, S.2.

184 Ebd., S. 2.

185 Conring, Hermann: De Aerario boni Principis (1663); vgl. Stolleis, Michael: Pecunia nervus rerum, S. 93.

186 Stolleis, Michael: Pecunia nervus rerum, S. $92 \mathrm{f}$.

187 Ebd., S.94f. 
indirekte Steuer). Zweitens schwächt sich der Legitimationsgrund „urgens necessitas“ - es muss eine dringende Not gegeben sein - allmählich ab; am Ende genügt der öffentliche Bedarf, um eine Belastung zu rechtfertigen. Und drittens geht man von einer ethischen Betrachtung zu einer empirischen über. Die in den Vordergrund rückende Empirie über ökonomische Gesetzmäßigkeiten und politische Interessen trieb ab Mitte des 17. Jahrhunderts bei den Merkantilisten einem Höhepunkt zu. ${ }^{188}$

\subsection{Die Leitbilder "gerechter" Besteuerung}

Die Autoren des frühmodernen Staates wollten mit ihren Vorschlägen die Obrigkeit auf die Einhaltung bestimmter Grundsätze und Regeln verpflichten. Dabei griffen sie zurück auf rechtliche und wirtschaftliche Erkenntnisse und auf ihre empirischen Erfahrungen über das Obrigkeitsverhalten. In den Schriften schlug sich dies in dem Versuch nieder, der landesherrlichen oder staatlichen Tätigkeit auf dem Gebiet der Finanzen Richtung, Ziel und Maß zu geben. ${ }^{189}$ Dabei war die Formulierung von wirtschaftlich begründeten, individuellen und gesamtwirtschaftlichen Prinzipien bestimmend. Um die landesherrliche Zugriffsbefugnis zu begrenzen, gilt das Prinzip vom Vorrang eigener Gütereinnahmen (Vorrang der Kammergefälle). Ergänzt wird dieses Prinzip durch die Formulierung und Präzisierung der Regel von der „necessitas“, dem Notcharakter der Steuern. Dieser ursprünglich materiell verstandene Begriff erfährt im Laufe der Zeit eine Veränderung: Aus einer als ethisch-christlich bewerteten bzw. existenziell verstandenen Not wird eine relative Not, die Notwendigkeit der Erfüllung aller möglichen selbst gesetzten Regierungsaufgaben. ${ }^{190}$ Die Folgen für das Verständnis der Steuer sind weitgehend:

Indem die Autoren vom Merkmal der „von außen“ kommenden materiellen Not als Rechtfertigungsvoraussetzung der Steuererhebung abrücken, geben sie auch die Vorstellung von der Steuer als einer notwendig zeitlich beschränkten, temporären Einrichtung preis. Aus der „extraordinären Hilfe“ wird die „ordentliche Steuer“ ${ }^{191}$

Mit Beginn des 17. Jahrhunderts bemerken die Autoren die Veränderungen in den fürstlichen Haushalten, die aufgrund eines gestiegenen Luxus der Hofhaltung zu

188 Ebd., S.96.

189 Schulz, Hermann: Das System und die Prinzipien der Einkünfte, S. 60.

190 Ebd., S. 64.

191 Ebd., S. 64. 
einer Zunahme von außerordentlichen Steuern führt. Statt neuer Steuern sollen die Ausgaben der Hofhaltung verringert werden. ${ }^{192}$ Appelle zur Sparsamkeit des Herrschers finden sich fast durchweg in der juristischen Literatur, wobei häufig auf den Bauernkrieg Bezug genommen wird. Ein ähnliches Bewusstsein für die kritische Lage des Gemeinwesens sowie Warnungen vor Neuerungen im Steuerwesen lassen sich erst wieder im Gefolge der Französischen Revolution feststellen. ${ }^{193}$ In den wiederkehrenden Mahnungen zur Mäßigung spiegeln sich die zeitweilig erdrückend hohen und ungleich verteilten Belastungen der Bevölkerung wider. ${ }^{194}$ In bildhafter Sprache plädieren die Autoren für eine Begrenzung der Steuerbelastung durch die Warnung nicht nur vor einer Besteuerung der Substanz, sondern auch vor negativen Anreizeffekten. ${ }^{195}$ Dies wird verdeutlicht durch plastische Vergleiche: „Doch wer zugleich das Fell abzieht / Bringt sich um kümfftigen Profit", reimte Karl Ferdinand Pescherinus 1718, und schon 1612 wusste Jacob Bornitz „Wann die Hüner gar geschlacht werden, so legen sie nimmer Eyer" ${ }^{196}$ Der Gedanke, die zulässige Gesamtsumme der Steuern zur Erfüllung der obrigkeitlichen Aufgaben zu bestimmen, führt zum Grundsatz des Übermaßverbotes. Der Fürst darf von seinem Recht auf Unterstützung nur im Rahmen des Bedarfs Gebrauch machen, soweit dies zur Erfüllung seiner anerkannten Herrschaftsaufgabe, des Schutzes vor inneren und äußeren Feinden, dient. ${ }^{197}$

\subsection{Die Diskussion um die gerechte Verteilung der Steuerlast}

\subsubsection{Maßstab und Art der Erhebung als Brennpunkte der Auseinandersetzung}

Die meisten Autoren des 17. Jahrhunderts halten als Maßstab zur Aufbringung der direkten Steuern eine Vermögensteuer für die sinnvollste und gerechteste Lösung. Dabei soll jedoch nicht das Vermögen als solches, sondern es sollen seine Erträge als Maßstab dienen. Einige Autoren wollen auch alle Gegenstände der

192 Schwennicke, Andreas: „Ohne Steuer kein Staat“, S. 124.

193 Ebd., S. $123 \mathrm{f}$.

194 Schulz, Hermann: Das System und die Prinzipien der Einkünfte, S. 66.

195 Scheer, Christian: Steuerpolitische Ideale, S. 160, mit Hinweis auf den Holländer Pieter De la Court in Anm. 25.

196 Zitate bei Homburg, Stefan: Allgemeine Steuerlehre, S. 35.

197 Schulz, Hermann: Das System und die Prinzipien der Einkünfte, S. 72. 
Steuer unterwerfen, die Nutzungen gewähren: Dazu gehören auch die Erträge aus Arbeitsleistungen und immateriellen Gütern. Hingegen sollen Gegenstände, die keinen Ertrag bringen, steuerfrei bleiben. ${ }^{198}$

Als Alternative zur Vermögensteuer wird häufig die Kopfsteuer diskutiert, die jedoch von der großen Mehrheit der Autoren als ungerecht und schädlich abgelehnt wird. ${ }^{199}$ Die Kopfsteuer gab es bereits im Altertum und unterwarf sogar die Besitzlosen dieser Steuer, die jeder Mensch in gleicher Höhe unabhängig von seinem Einkommen, Vermögen, Familienstand etc. entrichten musste. Sie war die inhumanste und unvollkommenste Art einer direkten Steuer, die jeden ohne Rücksicht auf seine Leistungsfähigkeit erfasste und deren unterdrückender Charakter als Einbuße an Freiheit empfunden wurde.

Kaiser Ludwig von Bayern führte 1342 die erste regelmäßige staatliche Kopfsteuer, den „Güldenen Opferpfennig" ein, der Juden auferlegt wurde. Nach Ludwigs Verordnung hatte sowohl jeder Jude, jede verwitwete Jüdin als auch jeder junge Jude über zwölf Jahre mit einem Vermögen von zwölf Gulden jährlich einen Gulden als Leibzoll an den König zu entrichten. ${ }^{200}$ Der Versuch, eine Kopfsteuer, die "poll tax“ in England einzuführen, endete mit einer großen Bauernrevolte im Jahr $1381 .{ }^{201}$

Seit Ende des 16. Jahrhunderts nahm in der politischen Praxis die Bedeutung der indirekten Verbrauchsbesteuerung stark zu. ${ }^{202}$ Neue territoriale Verbrauchsteuern blieben jedoch wenig ertragreich und in den wenigsten Fällen auf Dauer durchsetzbar, da die Untertanen immer häufiger die Erhebung und Abführung verweigerten. ${ }^{203}$ Die praktischen Schwierigkeiten bei der Einführung von Verbrauchsteuern auf umfangreiche Warenkategorien spiegelten sich auch in der politischen und juristischen Literatur wider. ${ }^{204}$

198 Ebd., S. $170 \mathrm{f}$.

199 Ebd., S. 172.

200 Schomburg, Walter: Lexikon, S. 136.

201 Dieses geschichtliche Ereignis hielt Margaret Thatcher nicht davon ab, die verhasste Kopfsteuer als Kommunalsteuer Ende der 1980er-Jahre in Großbritannien wieder einzuführen. Der Erfolg war, dass 18 Millionen Briten sich weigerten, die Steuer zu bezahlen, denn sie war ein glatter Verstoß gegen jede Version des Leistungsprinzips. Die Proteste gipfelten am 31. März 1990 in einer Demonstration mit rund 70.000 Teilnehmern in London, wobei es zu gewalttätigen Ausschreitungen kam. Letztlich war die „poll tax“ ausschlaggebend für die Krise und den Rücktritt der Regierung Thatcher. Vgl. Die Zeit vom 20.04.1990, Nr. 17.

202 Schwennicke, Andreas: „Ohne Steuer kein Staat“, S. 155.

203 Ebd., S. 158.

204 Vgl. hierzu die Ausführungen in Kapitel 5.2.2. 
Nach fast einhelliger Auffassung galt um die Jahrhundertwende von 16. zum 17. Jahrhundert die Luxussteuer als Idealsteuer. ${ }^{205}$ So hieß es bei den Autoren, dass Gegenstände, die nicht für den Lebensunterhalt notwendig seien, sondern überflüssigem Luxus dienten, vorrangig besteuert werden sollten. Lebensnotwendige Verbrauchsgegenstände sollten steuerlich unbelastet bleiben. ${ }^{206}$

\subsubsection{Zum Wandel im Verständnis der Steuerfreiheit von Adel und Klerus}

Im 17. Jahrhundert entwickelte sich ein neues Verständnis hinsichtlich der eingeschränkten Beteiligung - z. B. bei den (Reichs-)Türkensteuern - der Kirche und des Adels an den Steuern. In der Literatur wurde zunehmend die Untertaneneigenschaft auch des Adels betont und die soziale Schieflage der Steuerlastverteilung heftig kritisiert. Immer häufiger wurde ein funktionaler Zusammenhang zwischen der Steuerfreiheit und der Forderung nach Ausschluss von Kirche und Adel von gewinnorientierter Betätigung hergestellt; denn für die Immunität war jahrhundertelang Voraussetzung, dass der Steuerbefreite nicht am Handel teilnahm. Außerdem wurde die Ansicht vertreten, dass der Adel nur aufgrund seiner nicht mehr geleisteten Ritterdienste steuerfrei gewesen sei. ${ }^{207}$

Die Steuerfreiheit des Adels behandelte auch ein Gutachten aus dem Jahre 1641: Im römischen Recht lasse sich nirgends eine eindeutige Bestimmung finden, vielmehr ordne dieses an, dass Adel und Nichtadelige hinsichtlich der Lasten gleichbehandelt werden müssten. Im Übrigen bestehe auch kein allgemeines Gewohnheitsrecht, da der Adel in England, Spanien und in vielen deutschen Provinzen steuerpflichtig sei. ${ }^{208}$

Gegenüber der kirchlichen Steuerfreiheit blieben die meisten evangelischen Autoren kritisch eingestellt. Nach allgemeiner Auffassung müsse die Kirche zu den Reichs- und außerordentlichen Kriegssteuern beitragen. Auch die Besteuerung des persönlichen Vermögens der Kleriker in Notfällen blieb heftig umstritten. ${ }^{209}$

205 Schwenicke, Andreas: Ohne Steuer kein Staat, S. 162.

206 Ebd., S. $161 \mathrm{f}$.

207 Ebd., S. 180.

208 Ebd., S. 181.

209 Ebd., S. $176 \mathrm{f}$. 


\subsection{Zusammenfassung und Fazit}

Um die Jahrhundertwende vom 16. zum 17. Jahrhundert fand der wachsende Finanzbedarf des frühmodernen Staates seinen Niederschlag in der juristischen Steuerliteratur. Den Autoren erschloss sich die steigende Bedeutung der Geldeinkünfte für das Werden und Wachsen des Staates aus ihrer praktischen Tätigkeit. Der Wandel von der Natural- zur Geldwirtschaft und die neu empfundene Macht des Geldes hatten zu einem neuen Selbstverständnis dieser Zeit geführt. An die Stelle der Ablehnung jeglicher Steuern trat eine generelle Steuerrechtfertigungslehre; es genügte der öffentliche Bedarf, um eine steuerliche Belastung zu rechtfertigen. In den Schriften schlug sich der Versuch nieder, mit Leitbildern "gerechter" Besteuerung der Obrigkeit auf dem Gebiet Finanzen Richtung, Ziel und Maß zu geben.

\section{Zum Grundsatz der Allgemeinheit der Besteuerung}

Bereits im 16. Jahrhundert erhob Jean Bodin die Allgemeinheit der Steuerpflicht zur Voraussetzung der Besteuerung überhaupt. Er gründete seine Auffassung darauf, dass jede Besteuerung der Untertanen legitimiert und jede private Verwendung der Gelder der Obrigkeit ausgeschlossen sein müsse. Kasper Klock stützte das Gleichheitsprinzip auf das Naturrecht und forderte die Verminderung der Steuervorrechte des Adels und der Geistlichkeit.

Es entwickelte sich ein neues Verständnis hinsichtlich der eingeschränkten Beteiligung des Adels und der Kirche an den Steuern. Immer häufiger wurde ein funktionaler Zusammenhang zwischen Steuerfreiheit und der Forderung nach Ausschluss von Adel und Kirche von gewinnorientierter Betätigung hergestellt. Im Übrigen bestehe für den Adel auch kein allgemeines Gewohnheitsrecht, da dieser in anderen europäischen Ländern und in vielen deutschen Provinzen steuerpflichtig sei.

\section{Zum Grundsatz der Gleichmäßigkeit der Besteuerung}

Die gleichmäßige Durchführung der Besteuerung wurde in der Literatur als entscheidend angesehen. ${ }^{210}$ Seit Ende des 16. Jahrhunderts nahm die Bedeutung der indirekten Verbrauchsbesteuerung stark zu, was zu Unruhen in der Bevölkerung führte. In der Literatur schlug sich dies nieder, denn nach fast einhelliger Meinung wurde die Luxussteuer als ideale indirekte Steuer angesehen; entsprechend sollten die lebensnotwendigen Verbrauchsgegenstände steuerlich unbelastet bleiben. Zur Aufbringung der direkten Steuern wurde eine Vermögensteuer als die sinnvollste und gerechteste Lösung angesehen, wobei für die Besteuerung nicht das Vermö-

210 Schwenicke, Andreas: Ohne Steuer kein Staat, S. 169. 
gen selbst, sondern die Erträge als Maßstab dienen sollten. Dahinter verbarg sich einerseits die Kritik an der schematischen Besteuerung von Grundstücken nach veralteten Katastern, andererseits daran, dass Gegenstände, die keinen Ertrag brachten, steuerfrei bleiben sollten.

\section{Zum Prinzip der Besteuerung nach der Leistungsfähigkeit}

In allen juristischen Abhandlungen des 16. und 17. Jahrhunderts ist der Satz enthalten, dass die Untertanen nach ihrer Leistungsfähigkeit zu belasten seien; denn wer mehr verdiene, solle auch mehr zum öffentlichen Wohl beitragen. ${ }^{211}$

Kaspar Klock suchte nach einer gerechten, auf Leistung beruhenden Form der Besteuerung. Er wollte die Steuer nur auf Ertrag abwerfende Güter beschränken und näherte sich so dem Gedanken einer Einkommensteuer. Er forderte die Feststellung der Leistungsfähigkeit des einzelnen zu belastenden Objektes und war einer der Ersten, die die mit den Einnahmen zusammenhängende Ausgaben zur Feststellung des Reineinkommens zulassen wollten. Aus ethischen Gründen forderte er ebenso die Steuerbefreiung des Existenzminimums und warnte vor indirekten Steuern, da diese vor allem die Armen belasteten. Heutzutage wird Klock als Pionier der Finanzwissenschaft und als wichtigster Begründer der deutschen Steuerlehre benannt.

Dieses Buch wird unter der Creative Commons Namensnennung 4.0 International Lizenz (http://creativecommons.org/licenses/by/4.0/deed.de) veröffentlicht, welche die Nutzung, Vervielfältigung, Bearbeitung, Verbreitung und Wiedergabe in jeglichem Medium und Format erlaubt, sofern Sie den/die ursprünglichen Autor(en) und die Quelle ordnungsgemäß nennen, einen Link zur Creative Commons Lizenz beifügen und angeben, ob Änderungen vorgenommen wurden.

Die in diesem Buch enthaltenen Bilder und sonstiges Drittmaterial unterliegen ebenfalls der genannten Creative Commons Lizenz, sofern sich aus der Abbildungslegende nichts anderes ergibt. Sofern das betreffende Material nicht unter der genannten Creative Commons Lizenz steht und die betreffende Handlung nicht nach gesetzlichen Vorschriften erlaubt ist, ist für die oben aufgeführten Weiterverwendungen des Materials die Einwilligung des jeweiligen Rechteinhabers einzuholen.

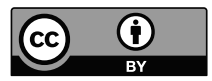

211 Ebd., S. $167 \mathrm{f}$. 